\title{
ANATOMÍA FLORAL DE LOS GÉNEROS Del COMPlejo Milla (Themidaceae)
}

\author{
Jorge Gutiérrez ${ }^{1,2}$, Teresa Terrazas ${ }^{3,5}$, Luis Hernández ${ }^{4}$ y Dorismilda Martínez-Cabrera ${ }^{1}$ \\ 1 Programa en Botánica, Colegio de Postgraduados \\ ${ }^{2}$ Unidad de Investigación en Sistemática Vegetal y Suelo, Universidad Nacional Autónoma de México \\ ${ }^{3}$ Departamento de Botánica, Instituto de Biología, Universidad Nacional Autónoma de México \\ ${ }^{4}$ Escuela de Biología, Facultad de Ciencias Naturales. Universidad Autónoma de Querétaro \\ ${ }^{5}$ Autor para la correspondencia: tterrazas@ibiologia.unam.mx
}

\begin{abstract}
Resumen: Se describe la morfo-anatomía floral de los géneros del complejo Milla (Themidaceae) con el objetivo de confirmar la presencia de un ginóforo en Dandya, de evaluar si existen caracteres anatómicos que permitan diferenciar a Dandya de los otros géneros del complejo (Behria, Bessera, Jaimehintonia, Milla, Petronymphe) y de hacer una interpretación evolutiva. Se estudiaron botones florales de diez especies del complejo Milla, los cuales se procesaron a través de la microtécnica de inclusión en parafina. Los resultados confirman la presencia de ginóforo sin médula en Dandya y con médula en los otros géneros estudiados. Además, Dandya se distingue de los otros géneros del complejo Milla por el estigma cerrado al terminar con sólo papilas externas, cavidades nectaríferas de forma astada con células epidérmicas rectangulares y filamento con 4 estratos de células parenquimatosas. Behria y Bessera comparten los estambres connados y la epidermis externa papilosa en el ovario. Los rasgos presencia de médula en el ginóforo, porcentaje de adnación del ovario al tubo floral entre 20-30\%, estigma abierto y forma discoidal de las cavidades nectaríferas se interpretan como la condición ancestral en el complejo Milla de donde se derivaron cambios exclusivos en Dandya.
\end{abstract}

Palabras clave: anatomía floral, Bessera, Dandya, ginóforo, Jaimehintonia, Milla, Petronymphe

\begin{abstract}
Floral morpho-anatomy of the Milla complex genera (Themidaceae) was studied with the aim to confirm the occurrence of a gynophore in Dandya, to determine if there are anatomical characters that allow to distinguish Dandya from the rest of genera of the complex (Behria, Bessera, Jaimehintonia, Milla, and Petronymphe), and to understand their evolution. Floral buds of ten species of the Milla complex were studied through the standard paraffin microtechnique. The results demonstrated the presence of a gynophore without pith in Dandya, while the other genera have a gynophore with pith. In addition, Dandya differs from the other genera of the complex by a closed-stigma with external papillae, nectary cavities of horned-shape with tabular epidermal cells, and filaments with 4-strata of parenchyma cells. Behria and Bessera share characters such as connated stamens and an ovary with the external epidermis papillose. Attributes like gynophore with pith, percentage of adnnation between ovary and floral tube of $20-30 \%$, stigma with an open ending, and nectary cavities with discoidal shape are probably ancestral conditions in the Milla complex, from which transformation of character states occurred mostly in Dandya.
\end{abstract}

Key words: Behria, Bessera, Dandya, ginóforo, Jaimehintonia, Milla, Petronymphe

os géneros Behria, Bessera, Dandya Jaimehintonia, Lilla y Petronymphe constituyen el complejo Milla en la familia Themidaceae (Pires et al., 2001; Pires y Sytsma, 2002; Gándara et al., 2009). Los géneros del complejo Milla comparten un conjunto de caracteres estructurales como son la presencia de cormo, las túnicas membranosas de fibras paralelas diminutas, el ovario estípitado por un ginóforo de longitud variable y la base del ovario adnada al tubo del perianto (Moore, 1953; Pires y Sytsma, 2002). Sin embargo, el tamaño del tubo floral, la connación de los filamentos, la presencia de ginóforo, la adnación de este último y del ovario con el tubo floral generó diversos cambios taxonómicos en los géneros del complejo Milla. Por ejemplo, Ingram (1953) consideró que Dandya purpusii presentaba un ovario no estípitado similar a Brodiaea y propone la combinación Brodiaea purpusii. Ese mismo año, Moore señala que esta especie si tiene un ginóforo pequeño y lo transfiere a Dandya. Mientras que en la descripción de las especies nuevas de Dandya no se menciona la presencia del ginóforo (Lenz, 1971; López-Ferrari y Espejo, 1992). Turner (1993) señala 
Jorge Gutiérrez, Teresa Terrazas, Luis Hernández y Dorismilda Martínez-Cabrera

que Jaimehintonia difiere de los géneros que conforman el complejo Milla sensu Moore (1953), porque el ginóforo y el ovario no están fusionados al tubo del perianto, interpretación que lo condujo a relacionar esta especie con Androsthephium, que pertenece al complejo Brodiaea sensu Moore (1953). Petronymphe (Moore, 1951) fue descrito sin presencia de adnación del ovario al tubo floral, situación que el mismo Moore (1953) rectifica años después. El género Behria fue segregado de Bessera con base en el tamaño del tubo floral y la connación de los estambres respecto a su longitud menor (Greene, 1886; Lenz, 1971). Además varios autores indican que los filamentos están connados en los miembros del complejo, pero su tamaño y forma difiere (Lenz, 1971; López-Ferrari y Espejo, 1992; Turner, 1993) o se ha generalizado sobre la adnación de la base del ovario con el tubo floral para todos sus miembros (Pires y Sytsma, 2002). A la fecha, existen varias interrogantes sobre los tamaños, grados de adnación y connación de los diferentes verticilos y la presencia y características del ginóforo para los géneros de este complejo, en particular para las cuatro especies de Dandya.

La utilidad de los caracteres anatómicos de la flor en la circunscripción en el nivel de familia y en la delimitación de géneros es ampliamente reconocida (Mogensen, 1969; Lenz, 1976; Fallen, 1985; Berg, 1996 y 2003; Endress, 1996; Rudall et al., 2002; González \& Rudall, 2003; Rudall, 2003; Rudall et al., 2003; Strange et al., 2004; Rudall et al., 2005; Wortley et al., 2005; Martínez-Cabrera, 2007; Sajo et al., 2010). Por tal motivo, se describe la anatomía floral de los seis géneros del complejo Milla sensu Gándara et al. (2009) con el propósito de: (1) confirmar si las especies de Dandya presentan ginóforo, (2) comparar las características del ginóforo de los géneros del complejo Milla, (3) identificar si existen caracteres de la anatomía floral exclusivos o compartidos entre los géneros del complejo y (4) examinar algunos rasgos florales a la luz de la filogenia más reciente del complejo Milla.

\section{Materiales y métodos}

Se realizó una revisión exhaustiva de la literatura y los herbarios (BH, CHAPA, ENCB, F, FEZA, FCME, GH, IEB, MEXU, RSA, TEX, UAMIZ, UC), así como de trabajo de campo para describir la morfología floral (Apéndice) y seleccionar material para desarrollar el estudio de la anatomía floral. Junto con los ejemplares de respaldo se recolectaron botones florales y flores en antesis de más de cinco individuos por especie de Bessera elegans Schult f., Dandya balsensis López Ferr. \& Espejo, D. hannibalii Lenz, D. thadhowardii Lenz, Milla biflora Cav. y M. magnifica H. E. Moore (Apéndice). Únicamente botones florales de Behria tenuiflora Greene, D. purpusii H. E. Moore, Jaimehintonia gypsophyla B. L. Turner y Petronymphe decora H. E. Moore fueron removidos de ejemplares de herbario (Apéndice).
Botones y flores en antesis fueron fijadas en formalinaácido acético- etanol (FAA) (Ruzin, 1999). Los botones florales removidos de ejemplares de herbario primero se hidrataron en $\mathrm{NaOH}$ al $5 \%$ por $12 \mathrm{~h}$ y posteriormente se fijaron en FAA por $24 \mathrm{~h}$. Todas las muestras fueron deshidratadas con alcohol ter-butílico (TBA) en un cambiador automático Leica (TP1020) y se procesaron según la microtécnica convencional de parafina (Ruzin, 1999). Los botones se cortaron transversal y longitudinalmente a $14 \mu \mathrm{m}$ de grosor con un micrótomo rotatorio Leica (RM2125RT); se tiñeron con safranina-verde rápido y montaron en resina sintética (Ruzin, 1999). La descripción se hizo siguiendo la propuesta de Dickison (1986).

El programa Mesquite versión 2.7 (Maddison y Maddison, 2009) fue utilizado para la optimización de los caracteres bajo el criterio de parsimonia. Se utilizó el árbol de consenso de Gándara et al. (2009), agregando sólo dos terminales al clado del género Dandya. Se seleccionaron seis caracteres de la anatomía floral. Dos de estos caracteres han generado cambios taxonómicos a lo largo de la historia del complejo Milla. Los seis caracteres seleccionados [connación de los estambres ausente (0), presente (1); epidermis externa del ovario no papilosa (0) o papilosa (1); cavidad nectarífera de forma discoidal (0) o astada (1); estigma abierto (0) o cerrado (1); porcentaje de adnación del ovario al tubo floral $20-30 \%(0)$, menor a $10 \%$ (1), ó $50 \%$ (2) y ginóforo con médula (0) o sin médula (1)], se trataron como no ordenados y a todos se les asignó el mismo peso.

\section{Resultados}

A continuación, se presenta la descripción morfológica (Figura 1) y anatómica (Figuras 2-5) para los géneros del complejo Milla. Se mencionan los nombres de los géneros o especies solamente en los casos donde existe variación intragenérica o infragenérica.

Morfología. Las flores se disponen de 2 a 30 en pseudoumbelas; con pedicelos de 0.25 a $8 \mathrm{~cm}$ de largo, por ca. $5 \mathrm{~mm}$ de diámetro, son filiformes, lisos, glabros o pubescentes; las flores son tubulares de forma campanulada, subcampanulada o hipocraterimorfa, algunas son fragantes de color blanco cambiando a púrpuras en la senectud, verdes-amarillentas, azules, púrpura, rojas o rosadas; el tubo del perianto es de 0.5 a $14 \mathrm{~cm}$ de largo, delgado en la base, expandido arriba, de 0.15 a 7 mm de diámetro; los tépalos tienen o no una línea longitudinal verde en el envés, en ocasiones cambiando a púrpura en la senectud, son oblongos a largamente ovados, glabros, con ápice obtuso los internos y ocasionalmente acuminados los externos, papilosos, tres a siete nervados, los exteriores de 0.3 a $3 \mathrm{~cm}$ de largo por 0.15 a $0.65 \mathrm{~cm}$ de ancho y los interiores de 0.3 a $3 \mathrm{~cm}$ de largo por 0.2 a $1 \mathrm{~cm}$ de ancho; los estambres son seis, libres, ensanchados en su base o connados por $0.2 \mathrm{~cm}$ o hasta $1.3 \mathrm{~cm}$, formando una corona; los filamentos son de color azul, blanco, rojo, rosa- 




Figura 1. Flores de los géneros del complejo Milla. A. Dandya thadhowardii (Gutiérrez 796). B. Bessera elegans (Gutiérrez 792). C. Petronymphe decora (L.O. Morrow BH-60-780). D. Jaimehintonia gypsophila (J.L. Reveal 3426). E. Milla magnifica (Gutiérrez 812). F. Milla biflora (Gutiérrez 809).

do o púrpura, de 0.1 a $5 \mathrm{~cm}$ de largo, glabros o papilosos; las anteras son amarillas, azules o verdes, en ocasiones singenéticas hasta poco antes de la senectud, lineares, basifijas, subasifijas o dorsifijas de 1 a $5 \mathrm{~mm}$ de largo por 0.3 a $1 \mathrm{~mm}$ de ancho; el ginóforo tiene de 0.5 a $13 \mathrm{~cm}$ de largo y es o no articulado; el ovario es trilocular, con múltiples óvulos, de $1 \mathrm{a} 12 \mathrm{~mm}$ de largo por 0.7 a $3.5 \mathrm{~mm}$ de ancho, introducién- dose al tubo entre el 15 y $100 \%$ de su longitud; el estilo es filiforme de 0.2 a $5 \mathrm{~cm}$ de largo; el estigma es linear, discoidal o trilobulado, cerrado o abierto y papiloso.

Anatomía. Perianto.- Tres tépalos externos e internos son anatómicamente indiferenciados (Figura 2A). La epidermis de los tépalos en ambas superficies es papilosa con cutícula estriada (Figura 2B, C), excepto en Milla y Petronymphe 


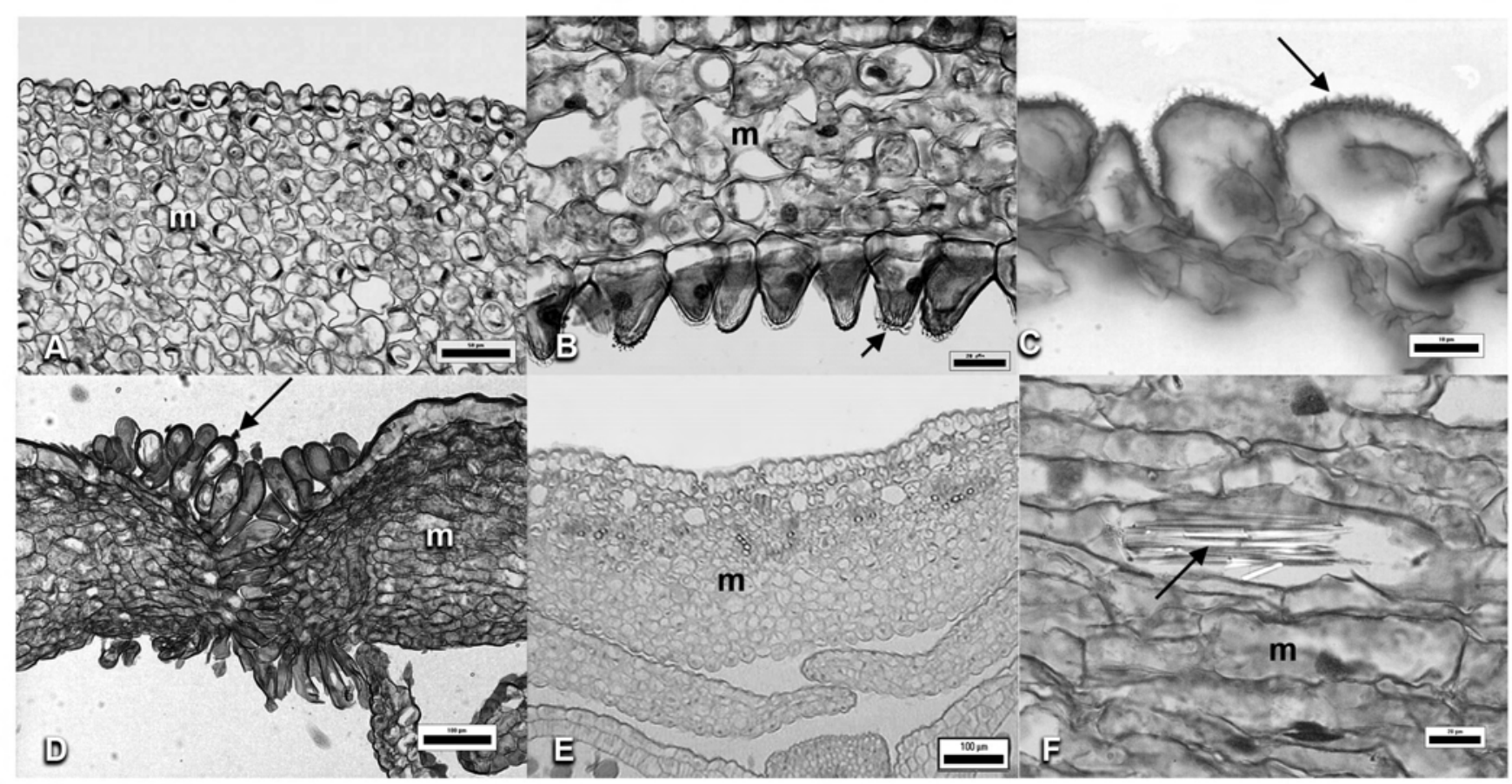

Figura 2. Perianto. A. Milla magnifica (Gutiérrez 812), epidermis papilosa en tépalo. B. Bessera elegans (Gutiérrez 799), epidermis papilosa con cutícula estriada (flecha) en tépalos. C. Jaimehintonia gypsophila (Hinton et al. 22461a), cutícula estriada (flecha) en epidermis de los tépalos. D. Dandya hannibalii (Gutiérrez 807), tricomas (flecha) en ápice de los tépalos. E. D. balsensis (Gutiérrez 839), tépalos. F. Milla biflora (Gutiérrez 808), rafidios (flecha) en mesofilo de un tépalo. Barra de $50 \mu \mathrm{m}$ en A; $20 \mu \mathrm{m}$ en B, F; de $10 \mu \mathrm{m}$ en C; de 100 $\mu \mathrm{m}$ en $\mathrm{D}, \mathrm{E} . \mathrm{m}=$ mesofilo.

con cutícula lisa; los estomas son anomocíticos y se encuentran en la superficie abaxial. En el ápice de los tépalos las papilas son más largas (Figura 2D). El mesofilo tiene parénquima no diferenciado, con un número de estratos celulares variable intragenéricamente (Cuadro 1, Figura 2E); hay rafidios en idioblastos embebidos en el mesofilo (Figura $2 \mathrm{~F}$ ) y 3 haces vasculares colaterales, excepto en $D$. purpusii, donde los 2 haces internos se dividen para formar un arco de 5 , con
5 ó 6 haces en M. biflora y hasta 7 ó 9 haces por tépalo en M. magnifica.

Androceo.- Los filamentos están adnados al tubo floral y se liberan asincrónicamente a una altura menor a $1 \mathrm{~mm}$ en Dandya (Figura 3A, E) y en Jaimehintonia. En Milla y en Petronymphe se aprecia un ensanchamiento en la base de la región donde se liberan los filamentos debido a que el parénquima es más abundante (Figura 3B, F). Únicamente

Cuadro 1. Número de estratos en tépalos, filamento, receptáculo, carpelo y óvulos en ovario en las especies del complejo Milla.

\begin{tabular}{|c|c|c|c|c|c|c|}
\hline \multirow[b]{2}{*}{ Especies } & \multicolumn{4}{|c|}{ Estratos } & \multirow{2}{*}{$\begin{array}{l}\text { Número } \\
\text { de óvulos }\end{array}$} & \multirow{2}{*}{$\begin{array}{l}\text { Adnación del ovario } \\
\text { al tubo floral (\%) }\end{array}$} \\
\hline & mesófilo & filamento & receptáculo & carpelo & & \\
\hline Dandya balsensis & $5-8$ & 4 & $12-14$ & $5-6$ & $12-15$ & $5-10$ \\
\hline D. hannibalii & $14-17$ & 4 & 4 & $5-6$ & $12-15$ & $<5$ \\
\hline D. thadhowardii & 5-8 & 4 & $9-11$ & $5-6$ & $12-15$ & $5-10$ \\
\hline D. purpusii & 9 & 4 & 6 & 8 & $18-21$ & $<5$ \\
\hline Bessera elegans & $14-16$ & 6 & $8-9$ & $9-10$ & $45-60$ & $20-30$ \\
\hline Behria tenuiflora & $6-8$ & 6 & $8-9$ & $6-7$ & $27-30$ & $20-30$ \\
\hline Jaimehintonia gypsophila & $13-15$ & 6 & 12 & 6 & $15-18$ & $20-30$ \\
\hline Milla biflora & $31-37$ & $10-12$ & 13 & $13-14$ & 66 & $40-50$ \\
\hline M. magnifica & $37-39$ & $9-10$ & 13 & $13-14$ & $51-60$ & $40-50$ \\
\hline Petronymphe decora & $13-15$ & 6 & 10 & $8-9$ & $39-42$ & $20-30$ \\
\hline
\end{tabular}






Figura 3. Androceo cortes transversales y longitudinales. A-D, H-M, cortes transversales. A. Dandya thadhowardii (Gutiérrez 798), filamentos al liberarse. B. Milla biflora (Gutiérrez 808), base ensanchada de un filamento al liberarse (flechas). C. Bessera elegans (Gutiérrez 799), estambres connados. D. Bessera elegans (Gutiérrez 799), filamentos al liberarse de la connación. E-G. Cortes logitudinales. E. Dandya balsensis (Gutiérrez 805), filamento a nivel de ovario. F. Milla magnifica (Gutiérrez 812), filamento con base ensanchada. G. Bessera elegans (Gutiérrez 799), estambres connados a nivel del ovario. H. Bessera elegans (Gutiérrez. 799), filamento ovoide. I. Milla biflora (Gutiérrez 808), filamento elíptico. J. Petronymphe decora (Moore 6937 bis), filamento ovoide-orbicular. K. Milla biflora (Gutiérrez 808), haz vascular bicolateral, xilema (flechas) entre floema. L. Dandya hannibalii (Gutiérrez 805), anteras tetrasporongiadas. M. D. hannibalii (Gutiérrez 805), epidermis de la antera con cutícula estriada (flecha) y endotecio. Barra de $300 \mu \mathrm{m}$ en A-G, de $100 \mu \mathrm{m}$ en H, I; de $50 \mu \mathrm{m}$ en J; de $20 \mu \mathrm{m}$ en $\mathrm{K}$; de $250 \mu \mathrm{m}$ en $\mathrm{L}$; de $10 \mu \mathrm{m}$ en M. en=endotecio, es=estilo, esc= estambres connados, fi=filamento, fl=floema, hv=haz vascular, ov=ovario, te=tépalo, $\mathrm{tf}=$ tubo floral 


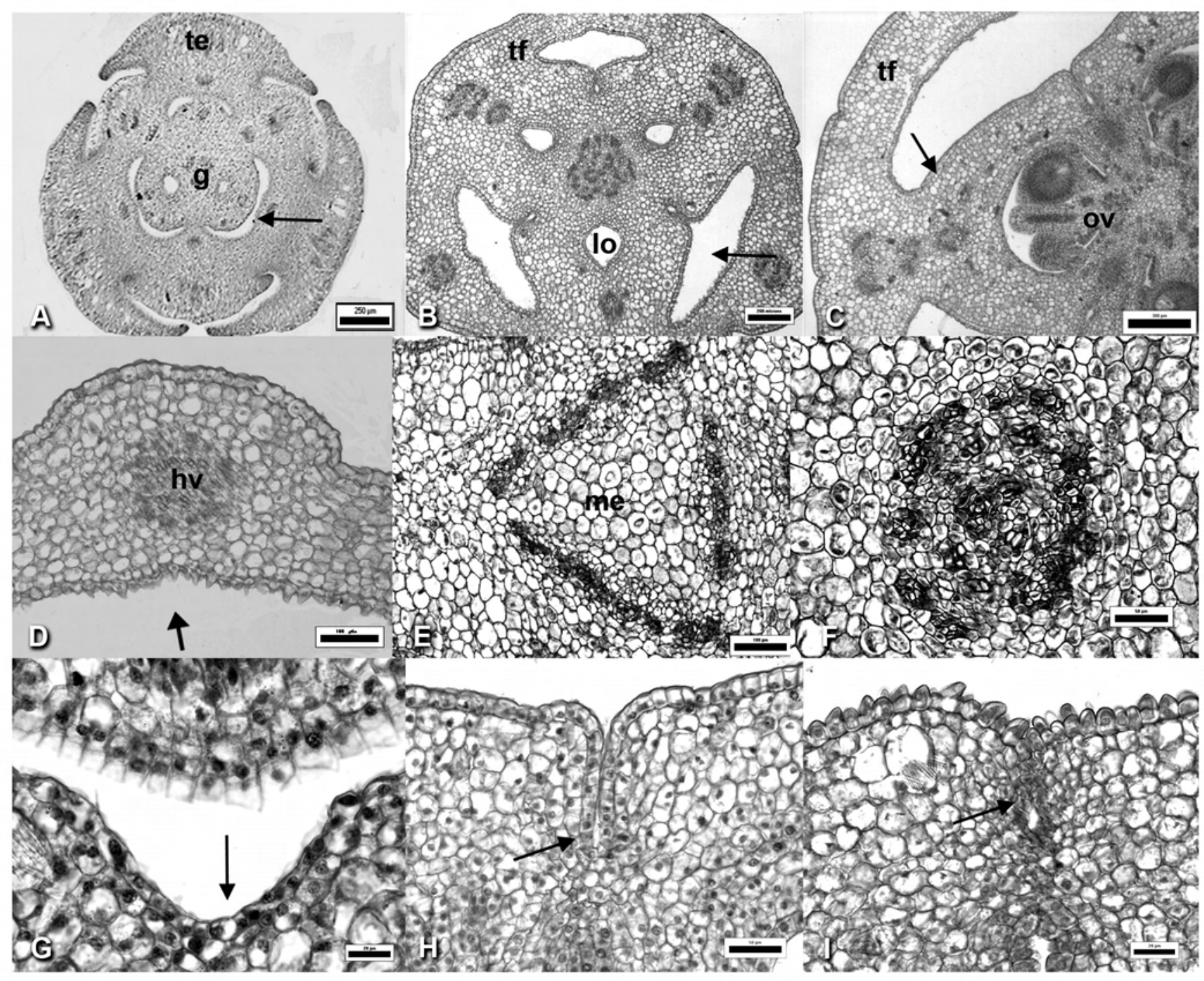

Figura 4. Ginóforo y nectario, cortes transversales. A. Dandya thadhowardii (Gutiérrez. 798), receptáculo mostrando base de tépalos, nectario (flecha) y ginóforo adnado al tubo del perianto. B. Bessera elegans (Gutiérrez 802), base el ovario adnada al tubo floral, nectarios septales (flecha). C. Milla magnifica (Gutiérrez 812), ovario y óvulos, con adnación del ginóforo y nectario septal (flecha). D. Bessera elegans (Gutiérrez 802), epidermis interna (flecha) del receptáculo papilosa. E. Milla magnifica (Gutiérrez 812), ginóforo con médula. F. Dandya balsensis (Gutiérrez 807) ginóforo sin médula. G. D. thadhowardii (Gutiérrez 798), nectario con pared recta. H. Milla magnifica (Gutiérrez 812), nectario con pared recta (flecha). I. Bessera elegans (Gutiérrez 802), nectario con pared papilosa (flecha). Barra de 250 $\mu \mathrm{m}$ en A; de $200 \mu \mathrm{m}$ en B; de $300 \mu \mathrm{m}$ en C; de $100 \mu \mathrm{m}$ en D, E; de $50 \mu \mathrm{m}$ en F, H; de $20 \mu \mathrm{m}$ en G, I. g=gineceo, hv=haz vascular, lo=lóculo, $\mathrm{me}=$ médula, ov=ovario, te=tépalo, $\mathrm{tf}=$ tubo floral.

los estambres están connados en Behria y Bessera (Figuras 1B, 3C, D, G). El tejido de connación está compuesto de parénquima y epidermis, y éste forma un tubo hasta de 13 $\mathrm{mm}$ de longitud que se prolonga hasta la parte media de los estambres (Figura 3G) en ambos géneros. Los filamentos son predominantemente redondos a orbiculares, vistos en sección transversal, pero de ovoides a orbiculares en Dandya hannibalii y Petronymphe y elípticos en Milla (Figura 3H-J); su epidermis es papilosa excepto en Dandya y Milla, y con la cutícula estriada. Bajo la epidermis hay de 4 estratos de células de parénquima en Dandya hasta 12 en Milla (Cuadro 1) y en el centro del filamento un haz vascular de tipo colateral o bicolateral exclusivamente en Milla (Figura $3 \mathrm{~K}$ ); las anteras son tetrasporangiadas (Figura $3 \mathrm{~L}$ ), con epidermis papilosa y cutícula estriada, excepto en $M$. magnifica con células epidérmicas rectangulares y cutícula lisa; el endotecio se constituye de un estrato con engrosamientos paralelos a sus paredes anticlinales (Figura 3M); además en la 


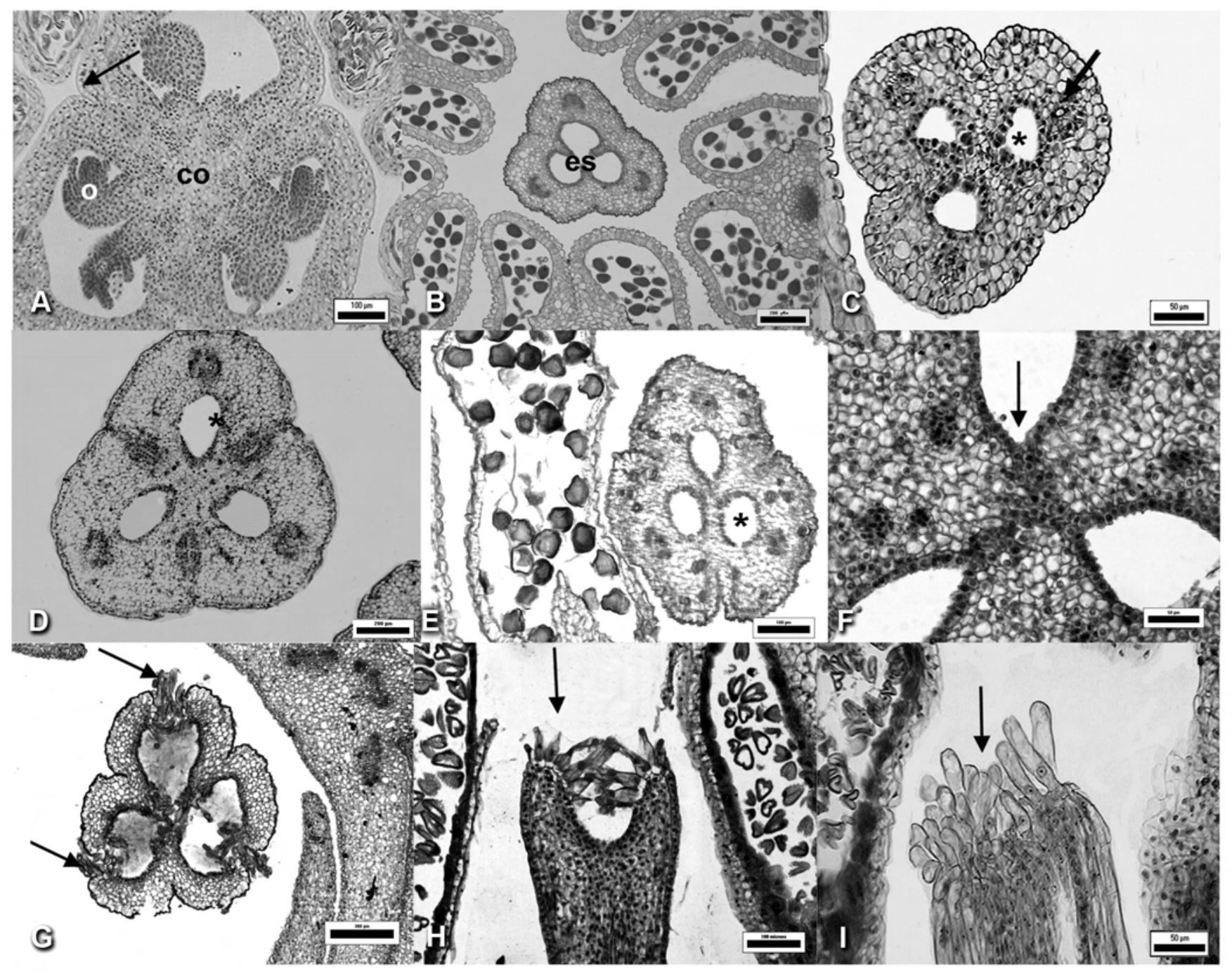

Figura 5. Gineceo, cortes transversales y longitudinales. A-G. Cortes transversales. A. Dandya hannibalii (Gutiérrez 807), ovario y nectario (flecha). B. Bessera elegans (Gutiérrez 799), anteras y estilo trilobado con las cavidades abiertas en el ápice. C. Dandya thadhowardii (Gutiérrez 798), estilo con cavidades triangulares (asterisco) y tejido vascular (flecha). D. Milla magnifica (Gutiérrez 812), estilo con cavidades ovoides (asterisco). E. Petronymphe decora (Moore 6937 bis), estilo trilobado, cavidad redonda. F. Milla magnifica (Gutiérrez 812), tejido transmisión (flecha). G. Milla biflora (Gutierrez 808), tejido de transmisión en estigma (flechas). H-I, cortes longitudinales. H. Bessera elegans (Gutiérrez 802), estigma papiloso (flecha). I. Dandya thadhowardii (Gutiérrez 798), estigma papiloso (flecha). Barra de $100 \mu \mathrm{m}$ en A, E, H; de $200 \mu \mathrm{m}$ en B, D; de $50 \mu \mathrm{m}$ en C, F, I; de $300 \mu \mathrm{m}$ en G. co=columna, es=estilo, o=óvulo.

parte dorsal hay tejido conectivo y en el centro de la tétrada de los esporangios un haz vascular colateral, con excepción de Milla con el haz vascular bicolateral.

Gineceo.- El receptáculo tiene células epidérmicas (Figura 4A), en ambas superficies, de forma rectangular en Dandya, pero en los otros géneros la epidermis externa es de forma cuadrada, vista en corte transversal y la interna papilosa en Behria, Bessera, Jaimehintonia y Petronymphe (Figura 4D), cutícula externa es lisa y la interna estriada solamente en Jaimehintonia. Bajo la epidermis se encuentran varios estratos de células de parénquima (Cuadro 1), 6 haces vasculares colaterales, excepto en Petronymphe con 9 haces y abundantes idioblastos con rafidios. El ovario es súpero, trilocular sostenido por un ginóforo con médula, excepto en Dandya sin médula (Figura 4E, F). El ginóforo está adnado parcialmente al tubo del perianto en su porción más basal en Dandya (Cuadro 1), o bien el ginóforo y la base de la porción fértil del ovario están adnados parcialmente al tubo floral en Behria, Bessera, Jaimehintonia y Petronymphe o adnado hasta la mitad de la porción fértil del ovario en $M i$ - 

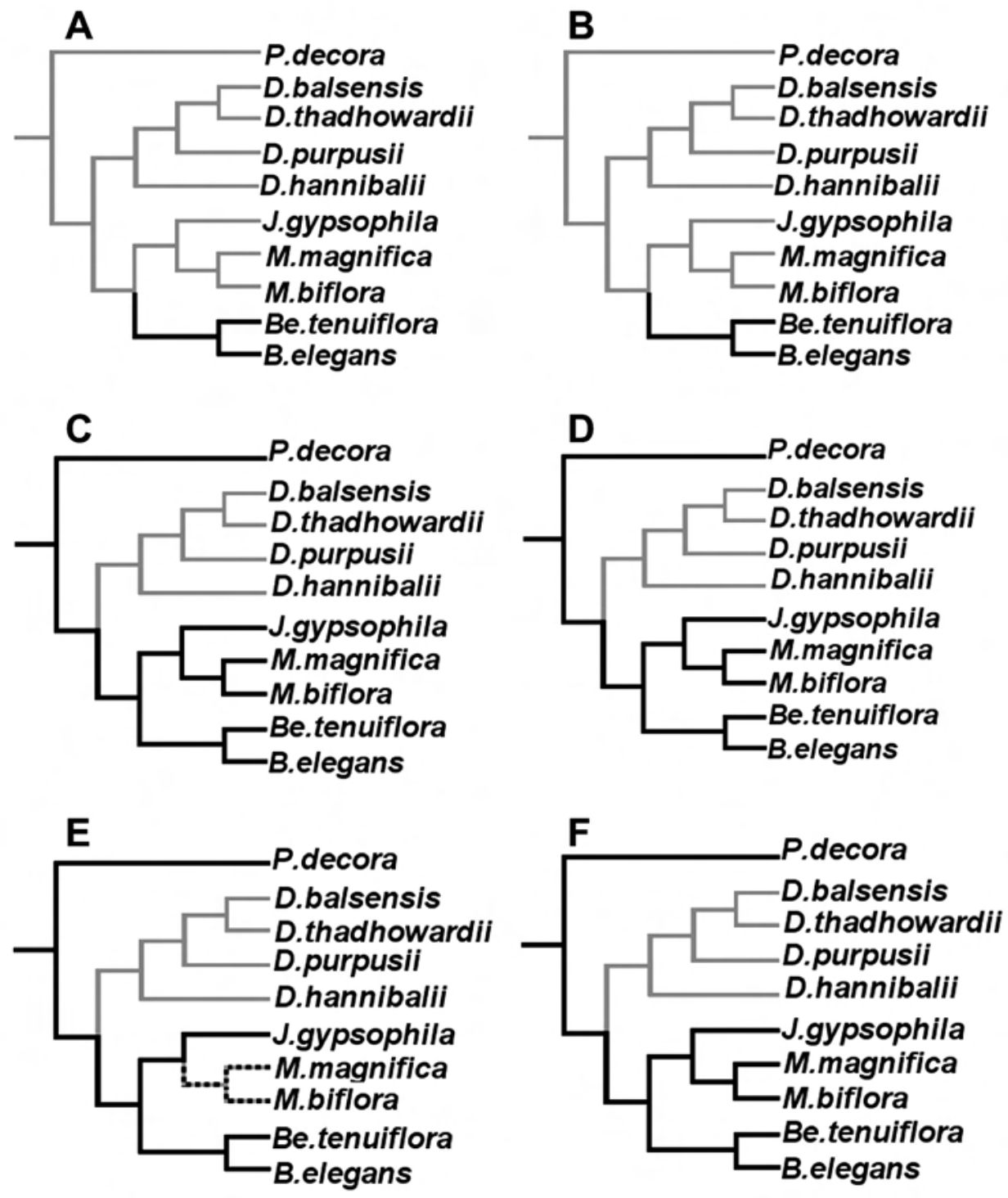

Figura 6. Reconstrucción de la evolución de seis caracteres de la anatomía floral de los miembros del complejo Milla. A. Connación de los estambres: ausente (blanco), presente (negro). B. Epidermis externa del ovario no papilosa (blanco), papilosa (negro). C. Forma de las cavidades nectaríferas en sección transversal: discoidal (negro), astada (blanco). D. Terminación del estigma: abierta (negro), cerrada (blanco). E. Porcentaje de adnación del ovario respecto al tubo floral: 20-30\% (negro), menos del 10\% (gris), mayor 40\% (punteado). F. Presencia de médula en ginóforo (negro), ausencia (blanco).

lla (Figura 4A-C, Cuadro 1). En todos los géneros, hay 3 cavidades nectaríferas de forma discoidal, pero astada en Dandya (Figura 4A-C). Los nectarios septales están formados por las células epidérmicas no papilosas de forma rectangular y con cutícula lisa en Dandya y Milla (Figura 4G, H) o papilosas con cutícula estriada en Behria, Bessera, Jaimehintonia y Petronymphe (Figura 4I). Las epidermis externa e interna del ovario es no papilosa en Dandya, Jaimehintonia, Milla y Petronymphe, pero papilosa la externa y no papilosa la interna en Behria y Bessera. Comúnmente, hay un haz vascular colateral dispuesto al centro de cada carpelo, excepto Jaimehintonia con 2 más ventrales formando un arco en cada carpelo. El número de estratos celulares en el carpelo es variable (Cuadro 1) así como los idioblastos con rafidios; la placentación es axilar, la placenta en forma de "Y", vista en corte transversal (Figura 5A). La columna está también formada por células de parénquima e idioblastos con rafidios. Hay un número variable de óvulos de 12 a 20 en la mayoría de los géneros, excepto en Milla con más de 50 óvulos; los óvulos son anátropos, crasinucelados y bitégmicos. El estilo es trilobado y abierto (Figura 5B) con epidermis papilosa y cutícula estriada, excepto en Dandya 
con epidermis no papilosa y cutícula lisa solamente en $D$. hannibalii. El estilo tiene tres cavidades de forma triangular en Dandya, de orbicular a triangular en Behria y Bessera, de redonda a ovoide en Jaimehintonia, ovoide en Milla y redonda en Petronymphe (Figura 5C-E). El tejido de transmisión se distingue por sus células pequeñas con citoplasma denso y núcleo evidente (Figura 5F). El tejido vascular está formado por 3 haces, uno al centro de cada lóbulo (Figura 5D). El estigma tiene epidermis papilosa que se continúa hasta la parte interna de las cavidades estilares en la mayoría de los géneros (Figura 5G, H), excepto en Dandya con las papilas sólo en la parte externa (Figura 5I), por lo que en el último se considera el estigma cerrado. Solamente las papilas en Behria, Bessera y Jaimehintonia tienen cutícula estriada. Estas papilas unicelulares se desarrollan al igual que en los otros géneros a partir de las células epidérmicas del tejido de transmisión.

Optimización de caracteres. Se presenta la optimización de seis caracteres de la anatomía floral en la figura 6. El género Dandya presenta el mayor número de cambios que se interpretan como novedades evolutivas (Figura 6).

\section{Discusión}

El género Dandya. Las cuatro especies de Dandya comparten los siguientes atributos: cuatro estratos de células de parénquima en el filamento, cavidades nectaríferas de forma astada con células epidérmicas rectangulares, ginóforo sin médula, estigma con papilas dispuestas externamente. Sin embargo, existen caracteres que permiten diferenciar a las especies del género. Por ejemplo, D. hannibalii y $D$. purpusii difieren de $D$. balsensis y $D$. thadhowardii por tener 5 a 8 estratos en el mesofilo de los tépalos y de 9 a 11 estratos celulares en el receptáculo. Dandya hannibalii se distingue por los cuatro estratos celulares en el receptáculo y el ovario de forma elíptica. Por otro lado, D. purpusii se diferencia por presentar mesofilo de los tépalos con 9 estratos de células, 5 haces vasculares por tépalo en forma de arco, receptáculo con 12-14 estratos de células, mesofilo de los carpelos con 8 estratos y óvulos en número de 18 a 21.

Comparación entre géneros. Behria, Bessera, Jaimehintonia, Milla y Petronymphe anatómicamente son muy semejantes, pero se diferencian de Dandya por la presencia de un ginóforo con médula, estigma con papilas que se continúan hasta la cavidad estilar generando un estigma abierto, cavidades nectaríferas en forma de disco y porción fértil del ovario adnada al tubo del perianto. Particularmente, Behria y Bessera se diferencia anatómicamente por la epidermis papilosa del ovario. Sólo comparte con Dandya la forma redonda orbicular del filamento, con Jaimehintonia y Petronymphe la epidermis papilosa del filamento y la porción del ovario adnada al tubo del perianto y únicamente con $P e$ tronymphe la epidermis papilosa del receptáculo.

Milla se distingue del resto de los géneros por tener hasta la mitad del ovario adnado al tubo floral, la presencia de 3040 estratos celulares compactos en el mesofilo de los tépalos, de 9 a 12 estratos de células en el filamento, un haz vascular bicolateral en el conectivo de la antera y de 50 a 66 óvulos. M. magnifica se diferencia de $M$. biflora por la cutícula lisa en la epidermis de los tépalos, la forma del filamento y la cutícula en la epidermis de las cavidades nectaríferas.

Jaimehintonia comparte con Milla y Petronymphe la presencia de una flor hipocraterimorfa aunque en los dos últimos géneros el tubo es de mayor tamaño, además comparten la epidermis interna del receptáculo papilosa con $B e-$ hria y Bessera. Al igual que Dandya, Milla y Petronymphe presentan la cutícula lisa en la epidermis del ovario. Petronymphe tiene semejanza anatómica con Jaimehintonia, pero se diferencia de éste por la cutícula estriada de la epidermis de los tépalos, por la forma elíptica de los filamentos en corte transversal, la epidermis interna papilosa del receptáculo y cutícula lisa de la epidermis de las cavidades nectaríferas. Un carácter exclusivo de Petronymphe es la cutícula lisa en la epidermis de los filamentos.

Caracteres. Todos los géneros presentan ginóforo pero difieren en su tamaño. Dandya presenta el ginóforo más corto de los miembros del complejo y el más largo se encuentra en Milla. Pires y Sytsma (2002) mencionan adnación del ovario al tubo del perianto para el complejo Milla. En este trabajo se muestra que el ovario se fusiona al tubo floral a diferentes niveles. En Dandya presenta fusión en la parte más basal del ovario y varía entre menos del $10 \%$ en $D$. balsensis y $D$. thadhowardii hasta menos del $5 \%$ en $D$. hannibalii y $D$. purpusii, por lo que un estudio de desarrollo floral permitirá confirmar esta diferencia. En Behria, Bessera, Jaimehintonia y Petronymphe la adnación se distingue en todo el ginóforo y hasta un tercio de la porción fértil del ovario, mientras que en Milla la adnación también es en todo el ginóforo pero hasta la mitad del ovario. Un estudio del desarrollo floral permita esclarecer el momento y la forma en que se establece la adnación en los miembros del complejo Milla.

El gineceo en los géneros estudiados es tricarpelar y trilocular con placentación axilar. De acuerdo con la clasificación de Dahlgren y Clifford (1982) los nectarios son septales. Este tipo de nectarios es común en las monocotiledóneas (Rudall et al., 2003) y se ha registrado también en otras especies de Themidaceae (Daumann, 1970; Berg, 2003). Los óvulos bitégmicos de tipo anátropo presentes en los géneros del complejo Milla, son característicos en la familia Themidaceae, aunque pueden presentarse los campilótropos (Berg, 2003). De acuerdo con Dahlgren (1991) los óvulos anátropos son comunes en las angiospermas y, generalmente, se consideran como un estado plesiomórfico.

El estilo es abierto en los géneros estudiados, es decir, cuenta con tres canales estilares divididos por tejido de transmisión. Esta característica ya se había registrado en Triteleia y Brodiaea también de Themidaceae (Rudall, 
2002). Así mismo, las Amaryllidaceae y todas las Asparagales derivadas presentan estilos abiertos con excepción de Alliaceae (Rudall, 2002), algunas Orchidaceae (Kocyan y Endress, 2001; Rudall y Bateman, 2002) y muchos pastos (Li y You, 1991), que presentan estilos sólidos. El estigma en las especies de Dandya se caracteriza por ser pequeño y tener una superficie reducida donde únicamente se encuentran las células del tejido de transmisión diferenciadas en papilas unicelulares que le dan la apariencia de cerrado, característica que contrasta con los otros géneros del complejo Milla. El estigma trilobado es abierto y las papilas se continúan hasta la parte interna de las cavidades estilares, ampliando el estigma en Behria, Bessera, Jaimehintonia, Milla y Petronymphe, como también se ha registrado en algunos géneros de leguminosas (Turano et al., 1983; Shivanna y Owens, 1989). Seguramente la disposición está relacionada con las diferentes formas que los polinizadores pueden depositar el polen.

Los estambres son adnados en todos los géneros y los filamentos se liberan asincrónicamente a diferentes niveles del tubo floral. Esta asincronía seguramente es la responsable de que se interprete como una connación en forma de anillo, disco o copa. Nuestras observaciones sugieren que únicamente Behria y Bessera tienen connación en los estambres. A esta connación de los estambres se le conoce como corona y en Bessera finaliza en la región superior del ovario donde se encuentran los óvulos (Figura 5G), siendo mucho más corta en Behria. Posiblemente, ésta sea una tendencia para proteger el ovario o atraer a polinizadores específicos con picos o probóscides muy desarrollados. No se observó connación en Dandya, Jaimehintonia, Milla y Petronymphe como otros autores habían interpretado (Lenz, 1971; LópezFerrari y Espejo, 1992; Turner, 1993). El ensanchamiento que se observa en la base de los filamentos corresponde a la terminación de la adnación donde se aprecia la liberación de los filamentos del tubo floral (Figura 5F). Nuestras observaciones anatómicas deberán de confirmarse con el estudio ontogenético del androceo en los géneros del complejo. Así mismo los filamentos en estos últimos géneros son muy cortos, disponiéndose inmediatamente por debajo de las bases de los tépalos.

Los rafidios fueron la única forma cristalina observada en los géneros estudiados y es el tipo cristales más común en algunas familias de Asparagales derivadas (Prychid y Rudall, 1999; Rudall et al., 2003) y en la subtribu Oncidiinae (Sandoval-Zapotitla et al., 2010). Los rafidios son importantes para distinguir entre familias y la ausencia en algunas monocotiledóneas es considerado como un estado derivado (Dahlgren y Rasmussen, 1983).

Evolución de caracteres. La forma discoidal de las cavidades nectaríferas, el estigma abierto y la presencia de médula en el ginóforo son los rasgos que comparten la mayoría de los géneros del complejo Milla y se consideran la condición ancestral. Además, la adnación entre el ovario y el tubo floral se considera la condición ancestral cuando hay un porcentaje de adnación entre $20-30 \%$ con dos transformaciones, una disminución en el porcentaje de adnación en Dandya y un incremento de dicha adnación en Milla (Figura 6E). Dandya es el género con mayor número de cambios y varios de ellos se interpretan como novedades evolutivas. Behria y Bessera comparten la connación de los estambres y la epidermis externa del ovario papilosa (Figura 6A, B) y junto con los caracteres mencionados por Gándara et al. (2009) apoyan su relación de parentesco.

\section{Conclusiones}

Las cuatro especies de Dandya presentan un ginóforo al igual que los otros géneros del complejo Milla. Las dos especies estudiadas del género Milla fueron muy variables en su anatomía por ello es necesario estudiar un mayor número de especies y confirmar que los caracteres señalados ayudan separar a los géneros del complejo Milla. Un estudio sobre ontogenia floral permitirá entender el proceso de adnación entre la porción fértil del ovario y el tubo del perianto, así como las diferencias en adnación y connación de los estambres en los géneros del complejo Milla.

\section{Agradecimientos}

Se agradece a los curadores de los herbarios el permiso para remover material para el estudio anatómico; al Consejo Nacional de Ciencia y Tecnología la beca (202590) otorgada al primer autor para realizar estudios de posgrado, al Colegio de Postgraduados las facilidades prestadas para realizar el trabajo de campo y de laboratorio, a Carmen Loyola por la toma de flores en estudio y a Julio César Montero Rojas por el diseño gráfico.

\section{Literatura citada}

Berg Y.R. 1996. Development of ovule, embryo sac, and endosperm in Dipterostemon and Dichelostemma (Alliaceae) relative to taxonomy. American Journal of Botany 83:790-801.

Berg Y.R. 2003. Development of ovule, embryo sac, and endosperm in Triteleia (Themidaceae) relative to taxonomy. American Journal of Botany 90:937-948.

Dahlgren R. 1991. Steps toward a natural system of the dicotyledons: embryological characters. Aliso 13:107-165.

Dahlgren R. y Clifford H.T. 1982. The Monocotyledons: A Comparative Study. Academic Press. London.

Dahlgren R. y Rasmussen N.F. 1983. Monocotyledon evolution: characters and phylogenetic estimation. Evolutionary Biology 16:255-395.

Daumann E. 1970. Das Blütennektarium der Monocotyledonen unter besonderer Berücksichti gung seiner systematischen und phylogenetischen Bedeutung. Feddes Repertorium 80:463590.

Dickison W.C. 1986. Floral morphology and anatomy of Staphyleaceae. Botanical Gazette 147:312-326. 
Endress P.K. 1996. Diversity and Evolutionary Biology of Tropical Flowers. Cambridge University Press, Cambridge.

Fallen M.E. 1985. The gynoecial development and systematic position of Allamanda (Apocynaceae). American Journal of Botany 72:572-579.

Gándara E., Sosa V. y León de la Luz J.L. 2009. Morphological and molecular evidence in the delimitation of Bheria and Bessera, two genera of the Milla complex (Themidaceae). Boletin de la Sociedad Botánica de México 85:113-124.

González F. y Rudall P.J. 2003. Structure and development of the ovule and seed in Aristolochiaceae, with particular reference of Saruma. Plant Systematics and Evolution 241:223-244.

Greene E.L. 1886. Studies in the botany of California and parts of adjacent V. Bulletin of the California Academy of Sciences 2:125-154.

Holmgren P.K., Holmgren N.H. y Barnett L.C. 1990. Index Herbariorum. Part 1: The Herbaria of the World. $8^{\text {th }}$ edition. International Association for Plant Taxonomy. The New York Botanical Garden, New York.

Ingram J. 1953. A monograph of the genera Bloomeria and Muilla (Liliaceae). Madroño 12:19-27.

Kocyan A. y Endress P.K. 2001. Floral structure and development of Apostasia and Neuwiedia (Apostasioideae) and their relationships to other Orchidaceae. International Journal Plant Science 162:847-867.

Lenz W.L. 1971. Two new species of Dandya (Liliaceae) from Mexico and a reexamination of Bessera and Behria. Aliso 7:313-320.

Lenz W.L. 1976. The nature of the floral appendages in four species of Dichelostemma. Aliso 8:383-389.

Li L.B. y You L.R. 1991. Structure and development of stigmatic branches and style their relation to pollen tube growth in wheat. Acta Botanica Sinica 33:712-717.

López-Ferrari A.R. y Espejo-Serna A. 1992. Una nueva especie de Dandya (Alliaceae) de la Cuenca del Río Balsas, México. Acta Botánica Mexicana 18:11-15.

Maddison W.P. y Maddison D.R. 2009. Mesquite:a modular system for evolutionary analysis, version $2.7<\mathrm{http}$ ://mesquiteproject.org> (consultado 1 abril, 2010).

Martínez-Cabrera D. 2007. Revisión de la tribu Hamelidae (Rubiaceae) con un enfoque morfo-anatómico. Tesis doctoral, Colegio de Postgraduados, Montecillo, Estado de México. México. $180 \mathrm{pp}$.

Mogensen H.L. 1969. Floral ontogeny and an interpretation of the inferior ovary in Agave parryi. Canadian Journal of Botany 47:23-26.

Moore E.H. 1951. Petronymphe, a new genus of Amaryllidaceae. Gentes Herbarum 8:258-260.

Moore E.H. 1953. The genus Milla (Amaryllidaceae-Allieae) and its allies. Gentes Herbarum 8:262-294.

Pires J.C. y Sytsma K.J. 2002. A phylogenetic evaluation of a bio- systematic framework:Brodiaea and related petaloid monocots (Themidaceae). American Journal of Botany 89:1342-1359.

Pires J.C., Fay M.F., Davis W.S., Hufford L., Rova J., Chase M.W. y Sytsma K.J. 2001. Molecular and phylogenetic analyses of Themidaceae (Asparagales). Kew Bulletin 56:601-626.

Prychid C.J. y Rudall P.J. 1999. Calcium oxalate crystals in monocotyledons: A review of their structure and systematics. Annals of Botany 84:725-739.

Rudall P. 2002. Homologies of inferior ovaries and septal nectarines in monocotyledons. International Journal Plant Science 163:261-276.

Rudall P.J. 2003. Unique floral structures and iterative evolutionary themes in Asparagales: Insights from a morphological cladistic analysis. Botanical Review 68:488-509.

Rudall P.J. y Bateman R.M. 2002. Roles of synorganisation, zygomorphy and homeosis in floral evolution:The gynostemium and labellum of orchids and other lilioid monocots. Biological Review 77:403-441.

Rudall P.J., Bateman R.M., Fay M.F. y Eastman A. 2002. Floral anatomy and systematics of Alliaceae with particular reference of Gilliesia, a presumed insect mimic with strongly zigomorphyc flowers. American Journal of Botany 89:1867-1883.

Rudall P.J., Cunniff J., Wilkin P. y Caddick L.R. 2005. Evolution of dimery, pentamery and monocarpellary condition in the monocot familiy Stemonaceae (Pandales). Taxon 54:701-711.

Rudall P.J., Manning J.C. y Goldblatt P. 2003. Evolution of floral nectaries in Iridaceae. Annals Missouri Botanical Garden 90:613-631.

Ruzin S.E. 1999. Plant microtechnique and microscopy. Oxford University Press, New York.

Sajo M.G., de Mello-Silva R. y Rudall P.J. 2010. Homologies of floral structures in Velloziaceae with particular referente to the corona. International Journal of Plant Science 171:595-606.

Sandoval-Zapotitla E., Terrazas T. y Villaseñor J.L. 2010. Diversidad de inclusiones minerales en la subtribu Oncidiinae (Orchidaceae). Revista de Biología Tropical 58:733-755.

Schivanna K.R. y Owens S.J. 1989. Pollen-pistil interactions (Papilionoideae). En: Stirton C.H. y Zarucchi J.L. Advances in legume Biology. Monographs Systematic Botany Missouri Botanical Garden 29:157-182.

Strange A., Rudall P. J. y Prychid C.J. 2004. Comparative floral anatomy of Pontederiaceae. Botanical Journal of the Linnean Society 144:395-408.

Turano M.J., Baird L.M y Webster B.D. 1983. Characteristics of the stigma in chickpea. Crop Science 23:1033-1036.

Turner B.L. 1993. Jaimehintonia (Amaryllidaceae-Allieae), a new genus from northeastern Mexico. Novon 3:86-88.

Wortley A.H., Scotland R.W. y Rudall P.J. 2005. Floral anatomy of Thomandersia (Lamiales), with particular reference to the nature of the retinaculum and extranuptial nectaries. Botanical Journal of the Linnean Society 149:469-482. 
Jorge Gutiérrez, Teresa Terrazas, Luis Hernández y Dorismilda Martínez-Cabrera

Apéndice. Material examinado de los géneros del complejo Milla. De los ejemplares del colector Gutiérrez se recolectaron muestras de cinco individuos por sitio, pero a los ejemplares de respaldo se les asignó un número de colecta por sitio. Acrónimos de los herbarios revisados o donde se depositaron los ejemplares de acuerdo con Holmgren et al.,(1990). Los ejemplares utilizados para el estudio anatómico se marcan con asterisco.

Bessera elegans Schultes f. COLIMA: Mpio. Colima, A.R. López-Ferrari et al. 917 (UAMIZ). CULTIVADA. Bailey Hortorium, Lawrence s/n (BH). EDO. DE MÉXICO: Mpio. Amatepec, M. Flores-Cruz et al. 244 (UAMIZ), A. Espejo et al. 3351 (CHAPA, UAMIZ), A. Espejo et al. 5545 (UAMIZ), A. Espejo et al. 2483 (UAMIZ), J.A. López et al. 1452 (CHAPA). GUERRERO: Mpio. Chilpancingo, H.E. Moore Jr. 5144, 8123, 8836 (BH), J. Gutiérrez et al. 799 (CHAPA, FEZA)*, S.D. Koch et al. 79136 (CHAPA), J. Ceja et al. 887 (UAMIZ); Mpio. Taxco, R.B. Clark s/n (BH), R. Fernández N. 2569 (BH), Rojas et al. 6605 (UAMIZ), J. Gutiérrez 802 (CHAPA, FEZA)*. JALISCO: Mpio. Guadalajara, M. Andreasen et al. s/n (BH), C.G. Pringle 11195 (BH); Mpio.Tlapa de Allende, A. Bruneau 594 y Rafael Lira (BH), S. Zamudio R. 7882 (UAMIZ); Mpio.Tecolotlán, R. Acevedo et al. 1461 (CHAPA); Mpio Manantlán, H.H. Iltis et al. 28808 (CHAPA); Mpio. Villa Guerrero, A. Espejo et al. 4499 (UAMIZ). MICHOACÁN: Mpio. Uruapan: F. Ventura A. 2410 (ENCB), S. Zamudio y E. Pérez C. s/n (UAMIZ), A. Espejo et al. 6605 (UAMIZ); Mpio. Parícuaro, Rzedowski 34198 (CHAPA, ENCB); Mpio. Chinicuila, S.D. Koch 87148 (CHAPA). MORELOS: Mpio. Xochitepec, A. Espejo et al. 6114 (UAMIZ); Mpio. Tlayacapan, R. Cerros T. 7 (UAMIZ); Mpio. Amacuzac, A. Espejo et al. 3646 (UAMIZ); Mpio. Tilzapotla, A. Espejo et al. 3855 (UAMIZ). NAYARIT: Mpio. Tepic, D. Bates et al. s/n (BH), M. Flores C. et al. 550 bis (UAMIZ); Mpio. San Pedro Lagunillas, A.R. López-Ferrari et al. 1734 (UAMIZ); OAXACA: Mpio. Putla, E. Solano C. 447 (CHAPA, FEZA). SINALOA: Mpio. San Ignacio, R. Vega et al. 844 (CHAPA), P. Fryxell 3038 (CHAPA); Mpio. Concordia, A.R. López-Ferrari et al. 1530 (UAMIZ).

Behria tenuiflora Greene. BAJA CALIFORNIA SUR: Mpio. La Paz, D. M. Porter 409 (GH), A. J. Gilmartin 2146 (MEXU), 1983, Brandegee, $(\mathrm{GH})$, B. J. Hammerly s/n (GH), M. E. Jones s/n (GH), T.L. Burgués 6193 (ENCB)*, D.E. Breedlove 43363 (ENCB)*, T.L. Burges 6193 (MEXU), R. Moran 18850 (MEXU), A. Carter 3458 (MEXU).

Dandya balsensis López-Ferr. \& Espejo GUERRERO: Mpio. Cutzamala de Pinzón, A. Espejo 2481 y T. Chehaibar (UAMIZ); Mpio. Eduardo Neri, S. Peralta et al. 231 (FCME), M. Gual 260 (FCME), Campos y Castelo 56 (FCME), M. Luna Flores 43 (FCME); Mpio. Iguala, Campos y G. Velásquez 118 (FCME), J. Saldivar y Sánchez s. n. (FCME), Velázquez Toledo y Campos 63 (FCME). MICHOACÁN: Mpio. Huetamo de Nuñez, A. Espejo 2467 y T. Chehaibar (UAMIZ), J. C. Soto y E. Martínez 4047 (ENCB, MEXU y UAMIZ); Mpio. San Lucas, J. Gutiérrez 805 (FEZA)*. MORELOS: Mpio. Tlaquiltenango, A. Flores Castorena 1075 y D. Martínez Alvarado (UAMIZ, ENCB, IEB), J. Gutiérrez 797 (FEZA)*, J. Gutiérrez 839 (FEZA)*.

Dandya hannibalii L. W. Lenz. MICHOACÁN: Mpio. Mugica, T.M. Howard 67-64 (RSA); Mpio. Huetamo, J. Gutiérrez 807 (FEZA)*, J. Gutiérrez 813 e I. Escalante (FEZA)*.

Dandya purpusii (Brandegee) H. E. Moore COAHUILA: Mpio. Ramos Arizpe, C.A. Purpus s.n. (GH, UC), M.F. Robert y J. Passini 4675 (ENCB)*, J.A. Villarreal 3980 (TEX)*, J.M. Porter 11308 y J.T. Columbus (RSA)*.

Dandya thadhowardii L. W. Lenz. GUERRERO: Mpio. Iguala, T.M. Howard 64-74 (RSA), J. Gutiérrez 798 (FEZA)*, J. Gutiérrez 840 (FEZA), J. Gutiérrez 841 (FEZA)*, J. Gutiérrez 842 (FEZA)*; Mpio. Coyuca de Catalán, T.M. Howard 68-222 (RSA), J. Gutiérrez 804 (FEZA)*, J. Gutiérrez 844 (FEZA)*.

Jaimehintonia gypsophila B. L. Turner (TEX!). NUEVO LEÓN: Mpio. Aramberri, Hinton et al. 22461 (IEB)*, J. L. Reveal 3426 (TEX!), Hinton et al. s.n. (UAMIZ).

Milla biflora Cav. AGUASCALIENTES: Mpio. Aguascalientes, R. McVaugh 8732 (ENCB). CHIHUAHUA: Mpio. Basaseachi, J. Gutiérrez 808 (FEZA)*. DISTRITO FEDERAL: Cerro del Chiquihuite, M.L. Arreguín 324 (ENCB), Xochimilco, F. Soto J. 173 (ENCB), Xochitepec, M. García s/n (ENCB). DURANGO: Mpio. Canacatlán, D.J. Pinkava s/n (ENCB); Mpio. Durango, W.R. Anderson et al. 5201, 3572 (ENCB); Mpio. El Salto, A. May Nah 2351 (ENCB); Mpio. Súchil, S. González con S. Acevedo 1923 (ENCB), F. Chávez 18 (ENCB). EDO. DE MÉXICO: Mpio. Ajacapusco, A. Ventura A. 1965 (ENCB); Mpio. Calacoaya, F. Medellín. 374 (ENCB); Mpio. Coacalco, S. Pérez H. 96 (ENCB); Mpio. Ixtapan de la Sal, J.A. López et al. 1416 (CHAPA); Mpio. Otumba, A. Ventura s/n (ENCB); Mpio. Texcoco, M.T. Pulido 488 (CHAPA), R. Fernández N. 4249 (CHAPA). GUANAJUATO: Mpio. Guanajuato, R. Galván et al. 3127 (CHAPA); Mpio. San José Iturbide, J. Gutiérrez 360 (FEZA), E. Ventura y E. López 6959 (IEB). HIDALGO: Mpio. Zapotlán: M. Equihua 524 (ENCB); Mpio. Zempoala, A. Ventura A. 1768 (ENCB). MICHOACÁN: Mpio. Ciudad Hidalgo, G. Mick 168 (ENCB). PUEBLA: Mpio. Jolalpan, E. Guízar 1419 (ENCB); Mpio. Mazapiltepetec, F. Ventura A. 2350 (IEB). TLAXCALA: Mpio. Apizaco, E. Ruíz B. 47 (ENCB); Mpio. Tlaxco, A. Martínez-Bernal et al. s/n (CHAPA). ZACATECAS: Mpio. Río Grande, M. Rodríguez s/n (ENCB); Mpio. Villanueva, E.D. Enríquez 420 (CHAPA).

Milla magnifica H. E. Moore (BH!). GUERRERO: Mpio. Iguala, A. Espejo 5205 (UAMIZ), A. Espejo et al. 5205 (Bailey Hortorium), J. Gutiérrez y R. Muñoz 823 (FEZA), J. Gutiérrez 838 (FEZA)*; Mpio. Taxco, A. Espejo et al. 5215 (IEB, UAMIZ); Mpio. Teloloapan, J. Gutiérrez 812 e I. Escalante (FEZA, CHAPA)*, R. L. Dressler (GH), H.E. Moore 5974 (MEXU). Teloloapan, laderas cerca del Río los Sabinos, 36-36 km, carretera Iguala-Teloloapan, XI.1949 (BH!).

Petronymphe decora H. E. Moore. GUERRERO: Mpio. Chilpancingo, H.E. Moore 5105 (BH!), H.E. Moore (6937 bis) (GH, BH, MEXU, UC), L.O. Morrow BH-60-780 (BH)*. 